\title{
GEOGRAPHIC OBJECTS: THEORY OR TECHNOLOGY DRIVEN?
}

\author{
D. N. Pantazis ${ }^{\mathrm{a}}$, E. Lazarou ${ }^{\mathrm{a}}$, P. Stratakis ${ }^{\mathrm{a}}$, H. Gadolou ${ }^{\mathrm{a}}$, A. Koukofikis ${ }^{\mathrm{a}}$, M. Kassoli $^{\mathrm{a}}$

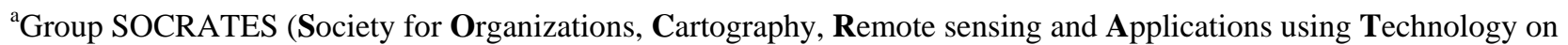 \\ Earth and Space), Surveying Engineering Department, School of Technological Applications, \\ Technological Educational Institution (TEI), Athens, Greece \\ Surveying Engineering Dept., School of Technological Applications, Technological Educational Institution , \\ Ag. Spyridonos, Athens, GR - dnpantaz@otenet.gr
}

Commission IV/8

\begin{abstract}
:
This article aims to compare the geo-graphic (spatial) objects in 2D (planar objects) proposed by CON.G.O.O. and ISO standards, and the ability of a number of GIS software to handle them. The results achieved so far showed that the actual technical possibilities are not always sufficient to support the complex spatial objects proposed by CON.G.O.O.
\end{abstract}

KEY WORDS: GIS, Cartography, Modelling, Data Structures, Database, Feature

\section{INTRODUCTION}

\subsection{The context and objectives}

In 1994, the conceptual formalism CONGOO (part of MECOSIG GIS design method), an acronym for the words CONception Geographique Orientee Object was proposed (Pantazis, 1994; 1996; 1997). The formalism includes an analytical classification of all possible geographic “vector” 2D objects that is possible to be used in spatial databases to represent real world in a digital 2D form. Almost 20 years later we try to see if all proposed objects are possible to be created by a number of existing GIS software.

\subsection{Structure of the article}

This article comprises eight sections and it is organised as follows: Section 2 reviews some basic concepts and definitions related to the spatial objects. In section 3 the MECOSIGCONGOO objects proposal is presented. Section 4 summarizes ISO proposal. Sections 5, 6, and 7 present the possibilities of spatial objects creations of ARC GIS, AutoCAD Map and one Open GIS: Quantum GIS. Section 8 presents the conclusions and further perspectives.

\section{GEO-GRAPHIC, GEOGRAPHIC, CARTOGRAPHIC, SPATIAL AND OTHER OBJECTS.}

The term geographic object is generic and includes objects having or not graphic representation (in a database). The list of European countries with their capitals in a excel (.xls) file could be called geographic objects. The term geo-graphic has been introduced by Pantazis $(1994 ; 1996 ; 1997)$ to define the objects having a graphic representation which are georeferenced in a coordinates system and /or a geodetic reference system on earth (geo-). The term spatial includes all the above as can be used to describe objects in other planets, in other "worlds" (e.g. the human body), having or not a graphic representation. The term cartographic object refers to all objects present in the cartographic surface of a map. Other terms also exist such as geoobjects, georeferenced objects, etc. A literature survey of relative terms is in progress.

\section{THE MECOSIG - CONGOO OBJECTS PROPOSAL}

CON.G.O.O. defines and distinguishes classes (= groups of one kind of objects) and objects. The term « class » here has not the same meaning as in O.O. languages. It must be understood as « set ", « group of objects », or the totality of one similar kind of object (e.g. forests). The description of an object is provided by properties usually called "attributes", behavioural characteristics usually called treatments and/or methods (= complex treatments), and structural characteristics concerning the topological and other types of relationships between the objects, classes, layers and sub-layers (see further for the terms «layer », " sub-layer »). We distinguish the following object categories (Fig. 1):

(i) The geo-graphic objects (GO). The geo-graphic objects are the objects having a graphic representation spatially / geo- referenced.

(ii) The non geo-graphic objects (NGO) concern all the other kinds of objects (alphanumerical, aerial photos or satellite images without geographic reference, video data, etc.).

Three categories are sufficient to cover the whole range of geographic objects at the conceptual level. These are the following: (i) The simple geo-graphic objects (SGO). We may consider three different sub-categories:

- type point (SGO, type P) | - type line (SGO, type L)

- type polygon (SGO, type PL)

(ii) The composed geo-graphic objects (CGO.). This kind of object stems from the union or the division of simple geo-graphic objects of the same type, belonging to the same or different classes. According to whether the SGO belong to the same class or not, we obtain HCGO (Homogeneous CGO) or ECGO (hEterogeneous CGO) respectively.

(iii) The complex geo-graphic objects (CXGO). This category is made of geo-graphic objects that stem from the union of different types (points + lines, lines + polygons, etc.) of geo-graphic objects (simple, composed or complex or a combination of them). The same distinction as above can be made between Homogeneous CompleX Geographic Objects (HCXGO) and hEterogeneous CompleX Geographic Objects (ECXGO).

This classification of the geographic objects combines in a very powerful and original manner the geometric and semantic characteristics of the geographic data and allows a clear distinction between the different kinds of geographic objects. The semantic 
power of those concepts allows a precise and specific characterization of each geographic object.

\section{THE ISO PROPOSAL}

ISO and OGC collaborate (as the TOCG - TC211\&OGC - coordination group) and agree to the same standards regarding the geographic information data model.

According to ISO, the Geographic Object includes the Geometric Object (GM_Object) and the Topological Object (TM_Object). The geometry classes for the Geometric Object are (Fig. 2):

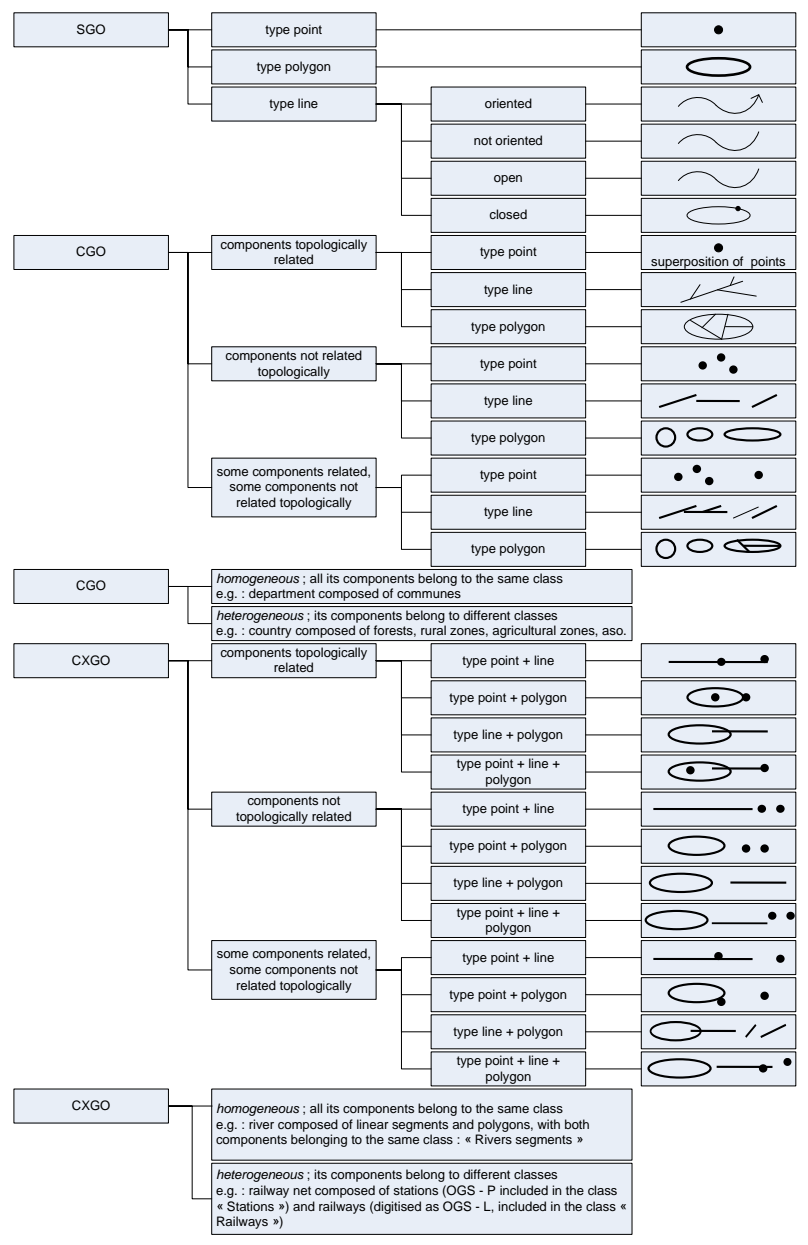

Figure 1. A summary of the different categories of geographic objects proposed by the CON.G.O.O. formalism.

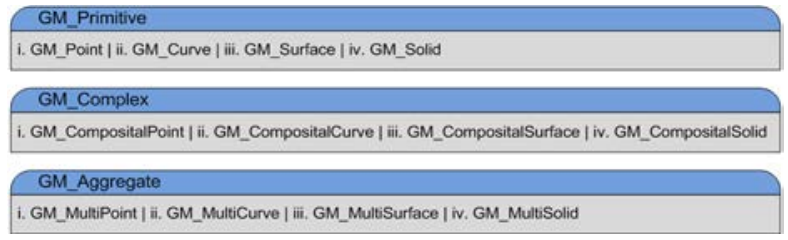

Figure 2. Geometry classes of the GM_Object (ISO, 2003)

The two standards that describe the spatial schema and the simple feature access of the geographic objects are:

(a) The ISO 19107:2003 - Spatial schema. ISO 19107:2003 specifies conceptual schemas for describing the spatial characteristics of geographic features, and a set of spatial operations consistent with these schemas. It treats vector geometry and topology up to three dimensions (www.iso.org). (b) The ISO 19125:2004 - Simple feature access. ISO 191251:2004 establishes a common architecture for geographic information and defines terms to use within the architecture. It also standardizes names and geometric definitions for Types for Geometry (www.iso.org).

The association between the CON.G.O.O. objects and the GM_objects proposed by the ISO (ISO, 2003) is presented in the next table (Table 1). Emphasising solely to the geometric dimension of the objects, the correlation of CON.G.O.O. and ISO objects is highlighted in the table below.

\begin{tabular}{|l|l|}
\hline CONGOO objects & GM_objects (ISO) \\
\hline SGO type point P & GM_Point (GM_Primitive) \\
\hline SGO type line L & GM_Curve (GM_Primitive) \\
\hline SGO type polygon/surface S & GM_Surface (GM_Primitive) \\
\hline & GM_MultiPoint \\
CGO type point P & (GM_Aggregate) \& \\
& GM_CompositePoint \\
& (GM_Complex) \\
\hline & GM_MultiCurve \\
CGO type line L & (GM_Aggregate) \& \\
& GM_CompositeCurve \\
& (GM_Complex) \\
\hline & GM_MultiSurface \\
CGO type polygon/surface S & (GM_Aggregate) \& \\
& GM_CompositeSurface \\
& (GM_Complex) \\
\hline CXGO & - \\
\hline
\end{tabular}

Table 1. The CON.G.O.O. objects associated to the GM_objects

\section{THE ARC GIS POSSIBILITIES IN COMPARISON WITH CONGOO OBJECTS}

\subsection{The case of ArcGIS software}

A Geodatabase in case of ArcGIS software contains datasets that include spatial features with attributes (Booth and MacDonald, 2006). The Geodatabase can also handle topology information, and can model behavior of features with rules on how features relate to one another. The feature classes are a set of features that are represented with one type of feature (point, line, or polygon). The ability of ARC GIS to handle CON.G.O.O. objects is presented below.

\section{SGO}

- Point. Point Shapefile or Point Feature Class (Stored in an ArcGIS Geodatabase model format). In ArcGIS software, Point is a geometric element defined by a pair of $\mathrm{x}$, $\mathrm{y}$ coordinates.

- Polygon. Polygon Shapefile or Polygon Feature Class (Stored in an ArcGIS Geodatabase model format). In ArcGIS software, Polygon is shape defined by one or more rings, where a ring is a path that starts and ends at the same point.

- Line. Polyline Shapefile or Polyline Feature Class (Stored in an ArcGIS Geodatabase model format). In ArcGIS software, Polyline is a shape defined by one or more paths, in which a path is a series of connected segments.

- Oriented: M (measure) values enabled. The direction of digitizing is stored in the SHAPE field.

$$
\begin{aligned}
& \square \text { Coordinates will contain } M \text { values. Used to store route data. } \\
& \square \text { Coordinates will contain } Z \text { values. Used to store } 30 \text { data. } \\
& \text { Enabling the } M \text {-values }
\end{aligned}
$$

- Not Oriented: M (measure) values disabled.

- Open: First and last vertex not coincident.

- Close: First and last vertex coincident. During digitizing, the last vertex must be snapped on the first one using 
an appropriate value of cluster tolerance (the cluster tolerance value is depended on the scale of the map).

\section{CGO}

Homogeneous CGO $\rightarrow$ In this case all the points OR Lines OR Polygons are stored in the same class and can be distinguished by using an Integer type field in the attributes table.

Heterogeneous CGO $\rightarrow$ In this case the different types of data are stored in separate classes (e.g. forests, rural zones, etc.).

ArcGIS in this case supports both Homogeneous and Heterogeneous CGOs.

- Topologically related

In ArcGIS software in order to control the topological relationships between any objects, the data need to be stored in Geodatabase format (Personal GDB, File GDB or ArcSDE Geodatabase) and a Topology Feature Class needs to be built and the topological rules to be set. The Shapefile format cannot handle topology operations.

\section{- CGO type Point}

Point Feature Class stored within a Feature Dataset of a Geodatabase. Feature Datasets are Collections of Feature Classes that share the same Coordinate System information. In this scenario we are able to ask from the software to ensure, for example, that points representing nodes in a pipe network junction will be coincident (superposition of points).

\section{- CGO type Line}

Polyline Feature Class stored within a Feature Dataset of a Geodatabase. In this scenario we are able to ask from the software to ensure, for example, that the branches of a stream or a road network are well connected to each to each other. Also we can group the polylines representing a river and its branches to form a single feature (one row in the database) by using the Merge command, or keep all the rows and add one more representing the whole of it using the Union command. This feature then is called Multipart Feature (Fig.3). In a case like this the analyst could calculate measures like the total length of all the parts that comprise it, even though the multipart feature is depicted as a single row in the database table (one ID).

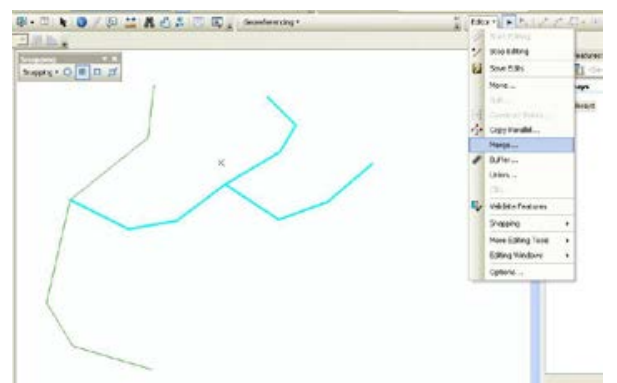

Figure 3. Merging Polyline features

\section{- CGO type Polygon}

Polygon Feature Class stored within a Feature Dataset of a Geodatabase. In this scenario we are able to ask from the software to ensure, for example, that the parcels of a city block are adjacent. Also we can group two or more polygons to form one feature in cases that we have for example an island complex, again by using the Merge command during the editing state, or keep all of them and add one more representing the whole of it using the Union command. This feature then is called Multipart Feature (Fig.4). In this case the analyst could calculate measures such as the total area or perimeter of all the parts that comprise it, even though the multipart feature is depicted as a single row in the database table (one ID).

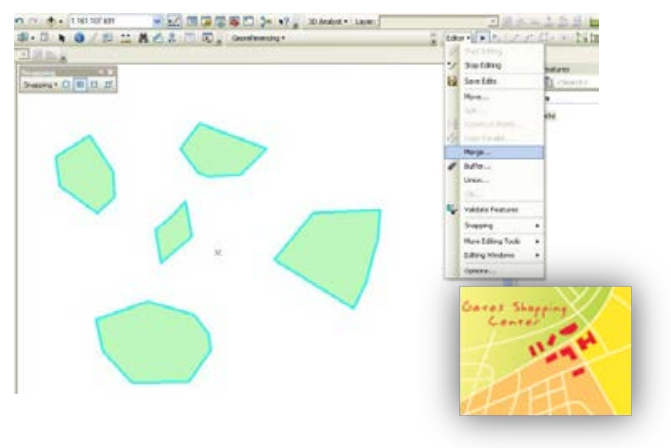

Figure 4. Multipart polygon feature (Source: ArcGIS Resource Center) Figure 9. Merging Polygon features

The Merge command combines selected features of the same layer into one feature. The features must be from either a line or a polygon layer. When merging, the user may choose which feature's attributes will be preserved during the operation. When a value is clicked in the Merge dialog box, the feature flashes on the map. The merge policy, if present, does not determine the value of the attributes in the merged feature (Fig.5). When features are not adjacent, a multipart feature will be created. For example, the user could merge the individual islands that make up Hawaii to create a multipart polygon feature.

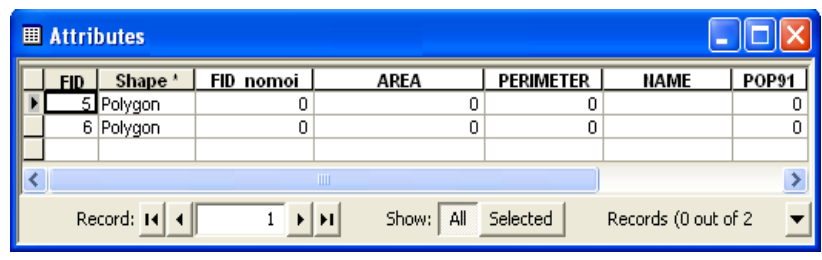
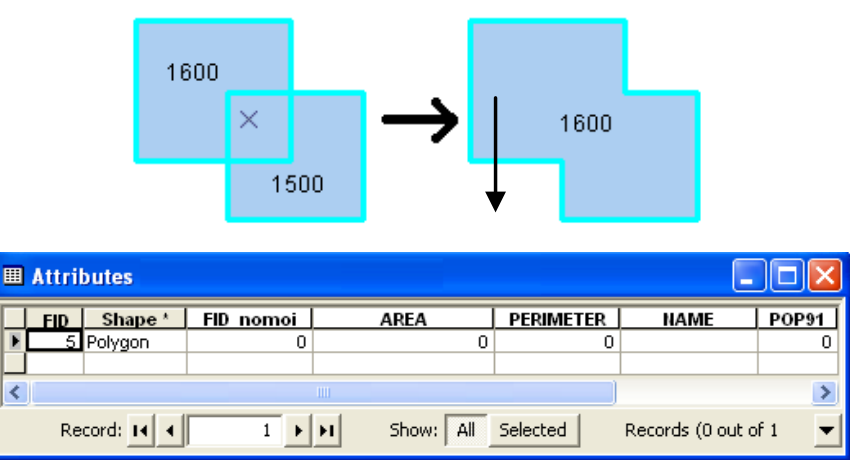

Figure 5. Example with Merge command

The Union command combines selected features into one new feature. Although the features may be from different layers, the layers must be of the same geometry type, either line or polygon. Union maintains the original features and their attributes. That is, the selected features are not deleted or edited during Union. The new feature is created in the current target layer with no attribute values. For example, with Union, the user can use selected parcels to create a polygon representing a neighbourhood crime prevention area.

Below is the new polygon feature in the target layer (crime prevention areas). The parcels are not affected by Union; they are simply hidden underneath the new polygon in this graphic (Fig. 6).

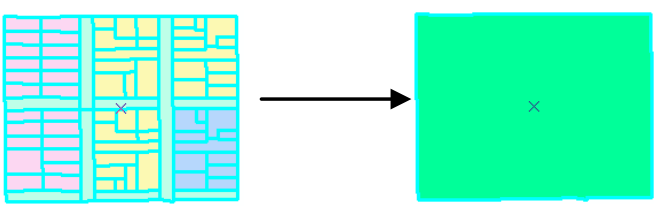


Figure 6. Example with Union command

The user can also create a multipart feature using the Union command by combining nonadjacent features from different layers.

Also there is possibility to construct a hole within a polygon ring (e.g. to create a lake within a polygon representing a forest), or even to auto-complete a polygon that already has one that we need to eliminate. We remind that for CONGOO formalism a polygon with hole(s) is considered as a CGO type polygon.

In the first case, the PLTS Create Hole tool creates holes in the selected target polygon layer (Fig. 7). This allows the user to create new polygons of a different feature class subtype for an area. For example, one can create a polygon of type Bamboo Cane in a Scrub Brush area because both are contained in the Grass layer.

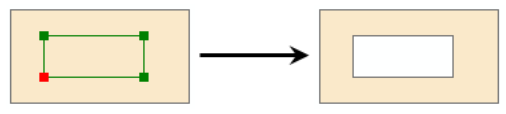

Figure 7. PLTS Create Hole tool result. Source: ArcGIS Desktop 9.3 Web help

In the second case, the user can use the Fill Hole tool to fill one, multiple, or coincident interior holes from selected polygon geometry. A coincident hole is the hole shared by layered polygons. There is one hole for each polygon (Fig.8), so if there are several polygons, there will be several holes called coincident holes.

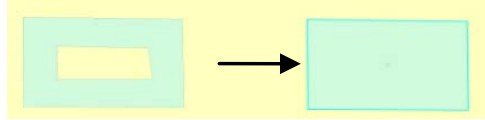

Figure 8. Fill Hole tool result. Source: ArcGIS Desktop 9.3 Web help

- Components of CGO Not Topologically related $\rightarrow$ Same as SGOs

- Some components of CGO have topological relations and/or some have not

o Point, Lines or Polygons

In a Point class one could have multiple features merged together occupying a single row in the attribute table along with isolated ones.

\section{CXGO}

Homogeneous CXGO $\rightarrow$ In this case two types of geometries appear in the same class. This is something that does not comply with the ArcGIS vector data model.

Heterogeneous CXGO $\rightarrow$ It is out of the software's logic to create a single object that can represent two or more types of geometry simultaneously. So, not in this case the software supports the complex features.

What ArcGIS can do to handle this is to store the different types of geometries in separate classes (Railways-Polyline, StationsPoint).

Concluding, ArcGIS by not supporting the complex features cannot apply techniques like the Buffering in multiple geometries in one operation. For example there is no way to create a buffer zone around a transportation network (edges \& nodes), or a hydrographic network (streams-lines and basins-polygons). The way that one could handle this is by creating buffer zones per geometry type (e.g. first for the edges then for the nodes) and after that to merge the buffers together to have a unique class holding the buffers of the network.

\section{THE AUTOCAD MAP POSSIBILITIES IN COMPARISON WITH CONGOO OBJECTS}

\subsection{The case of Autocad Map 3D}

Autocad Map is a GIS solution in data management and relating geographic features to databases (Karimi \& Akinci, 2010). Geospatial data in Autocad Map is organized as follows:

1.Feature: The spatial representation of a real-world entity, for example a specific road or an individual utility pole, that specifies the geometry and other properties of the feature.

2.Feature class: A category of features with rules that define the allowable data types, default values, and constraints for its member features. For example, one may have feature classes for a set of roads, utility poles, etc.

3.Schema: A collection of related feature classes.

4.Data store: A collection of feature data in a single storage location. SDF (Spatial Database File) data stores allow only one schema per file, but database stores such as Oracle or SQL Server can have multiple schemas. SHP files (Shape files) can include only one geometry type per file.

\subsection{Commands creating the CON.G.O.O. objects with Auto- cad 3D Map}

Steps to create new schemas:

- On the task pane of the graphical interface we select the tab "Map Explorer"

- Left click on "Schema"

- Create a new SDF file

- Enter name and path of the SDF file

- We browse the coordinate systems to select the coordinate system we work with

- Automatically appears the Schema Editor. We can add multiple features to our schema and decide the properties and the geometry of each feature

- The new schema created then appears

SGO creation:

- Point. Point Feature stored in a SDF file. On the task pane $>$ Right click on feature class $>$ Create New Point (Fig.9a).

- Line. Line Feature stored in a SDF file. On the task pane $>$ Right click on feature class $>$ Create New LineString (Fig.9a).

- Polygon. Polygon Feature stored in a SDF file. On the task pane $>$ Right click on feature class $>$ Create New Polygon (Fig.9a).

CGO creation:

- Not Topologically related Point. Different Point features occupying only one instance in the properties table. On the task pane $>$ Right click on feature class $>$ Create New MultiPoint (Fig.9b).

- Not Topologically related Line. Different Line features occupying only one instance in the properties table. On the task pane $>$ Right click on feature class $>$ Create New MultiLineString (Fig.9b).

- Not Topologically related Polygon. Different Polygon features occuping only one instance in the properties table. On the task pane $>$ Right click on feature class $>$ Create New MultiPolygon (Fig.9b).

- Topologically related Line. Different Line features occupying only one instance in the properties table. The object snapping of Autocad Map is used to relate the line features. 
On the task pane $>$ Right click on feature class $>$ Create New MultiLineString (Fig.9c).

- Topologically related Polygon. Different Polygon features occupying only one instance in the properties table. The object snapping of Autocad Map is used to relate the polygon features. On the task pane>Right click on feature class $>$ Create New MultiPolygon (Fig.9c).

- Polygon with hole. This CGO is created with Right click on feature class $>$ Create New MultiPolygon. We create the first polygon, then we create a second polygon inside the first. A hole is created (Fig.9c).

- Homogeneous CGO. Autocad Map is able to create homogeneous CGO because all objects belong to the same feature class.

- Heterogeneous CGO. Autocad Map is not able to create heterogeneous CGO because all objects do not belong to the same feature class.

CXGO creation:

Autocad map is not able to create CXGO. We can combine SGO or CGO objects to create a new object that looks like a CXGO (Fig.9d).

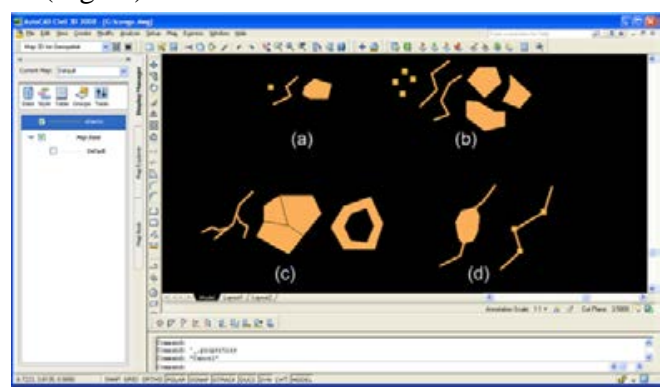

Figure 9. CONGOO objects created into AutoCad Map’s model

An example of the combination of SGO objects trying to represent a CXGO object (Fig.10) is the Greek railway network. The rails are represented as Lines and the stations as Points. The data table of the feature class "Railway Network" has common fields (ID) and non-common fields (STATION_NAME, LINE_LENGTH, etc). Each field must be configured to accept Null values because a station cannot have length and a line cannot have a phone number.

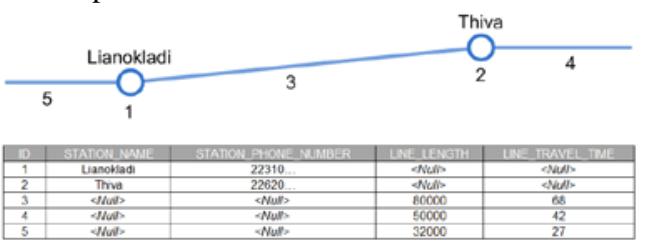

Figure 10. The Greek railway network as a combination of SGO 7 OPEN GIS POSSIBILITIES IN COMPARISON WITH CONGOO

\subsection{The case of Quantum GIS}

Quantum GIS (QGIS) is an Open Source Geographic Information System, licensed under the General Public License GNU. QGIS is an official project of the Open Source Geospatial Foundation. The software supports vector data in a number of formats as well as raster data and databases in various formats (www.qgis.org).

Each object (point, line, etc.) is stored in different classes or layers. The geometry is stored internally and is represented by the well-known binary (WKB) Geometry Format defined by the Open Geospatial Consortium (OGC) or as Geometry Engine Open Source (GEOS) geometry (geos.osgeo.org). The data model is according to the «OpenGIS Implementation Standard for Geographic information - Simple feature access» (OGC, 2010).

The CON.G.O.O. objects as supported in the QGIS software are presented in the following paragraph.

SGO

- $\quad$ type point: is represented by the QgsPoint class which is used to store $\mathrm{X}, \mathrm{Y}$ coordinates of the point and is created by the command Capture Point (Fig.11a).

- type line: is represented by the QgsPolyline class which includes a vector of points and is created by the command Capture Line. It is either oriented or not oriented, open or closed (Fig. 11 b,d,e,f).

- type polygon: is represented by the QgsPolygon class which is defined by one or more rings whereas first item of the list is outer ring, inner rings (if any) start from second item (Fig.11 c) and is created by the command Capture Polygon.

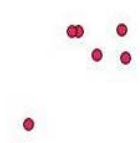

(a)

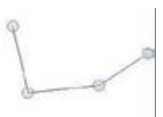

(d)

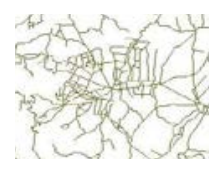

(b)

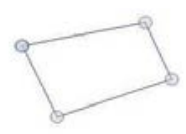

(e)

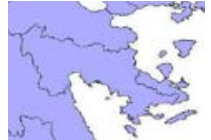

(c)

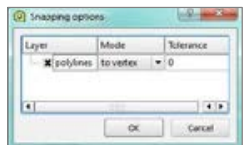

(f)
Figure 11. SGO objects in QGIS. (a) point, (b) line, (c) polygon, (d) open line, (e) closed line, (f) option for oriented or not lines.

\section{CGO}

QGIS supports CGO objects (points, lines, polygons) stored in the same class (Homogeneous CGOs) or in different classes (Heterogeneous CGOs). The CGO category is addressed by the QgsGeometryCollection of the QGIS software as follows:

- type point: is represented by the QgsMultiPoint class which is a collection of QgsPoints that share a common collection of attributes.

- type line: is represented by the QgsMultiPolyline class which is a collection of QgsPolylines that share a common collection of attributes.

- $\quad$ type polygon: is represented by the QgsMultiPolygon class which a collection of QgsPolygons that share a common collection of attributes.

CGOs can be topologically (or not) related establishing (or not) the necessary topological rules for each or some of the objects. Each object (e.g. a QgsMultiPolygon) is a unique feature stored in with a different object id and geometry and it can be created by the command Singleparts to multipart. The new attribute table includes all the attributes of the table of the objects that constitute it.

\section{CXGO}

The complex geo-graphic objects (CXGO) are not supported by the QGIS software. Each geographic object can only be addressed and stored in different classes either as SGO or CGO (e.g. point or multipoint).

Operations'examples 
An example of a CGO type polygon is the Prefecture of Cyclades in Greece, (represented as a QgsMultiPolygon object), that is a collection of QgsPolygons sharing a unique geometry and id. If we want to sum up the area of this object, this can be operated if it is considered as one polygon by adding a geometry column in the attribute table of the object. A buffer to CGO object is also possible and it will be created taking into account the centroid of the polygon. Note that if the polygons that form the CGO object are stored as QgsPolygons, the user must first merge them to a polygon (QgisMultiPolygon) by the command Singleparts to multipart.

In case we want to calculate the length of a CXGO (e.g. set of lines and polygons), in QGIS we have to calculate the total length of the polylines (stored in different classes) that constitute the CXGO. A railway network, for example, will be consisted of lines (representing the railway paths) and points (representing the stations). The length of the railway can be calculated as the sum up of the length of the polylines that form the railway paths.

\section{CONCLUSIONS AND FURTHER INVESTIGATION}

The Geographic Information Science - a recently used term which replaces in many cases the Geographic Information Systems- has been many times characterised as technology driven rather than theory, users, concepts or "something else" driven. Important software and equipment companies offer nowadays a variety of geo-sciences products and play an important role to the development in Geo-sciences business. On the other hand hundreds of Geo-sciences conferences, journals, symposiums present a vast number of new scientific achievements and applications. The primary question here is if there is any useful and important interaction, and if finally the end user is listened. Our research objective is to use an "easy" problem such as the creation of composite and complex geo-graphic/spatial objects, in order to test the possibilities of some GIS software. Current results show that the proposed by CONGOO formalism CXGO is not possible to be created by the GIS software analysed above. However evidence highlights that further investigation is needed and is already in progress concerning:

a) the exhausted presentation of proposed geo-graphic objects of the most popular standards.

b) the analytical literature review of all relative to "spatial objects" definition.

c) the test in creation and management composite and complex objects of other GIS software such as Smallworld Map Info, Manifold etc.

In addition to this, a series of problems related to attributes of spatial complex objects and spatial analysis process are investigated. The analysis identified the limited possibilities of the actual GIS software concerning, e.g. buffer operations in complex objects, relational algebra operations between composite and complex objects etc.

\section{ACKNOWLEDGEMENTS}

The research is supported and partially funded by the Technological Educational Institution (TEI) of Athens, Greece.

\section{REFERENCES}

ArcGIS Desktop 9.3 Web Help. http://webhelp.esri.com/arcgisdesktop/9.3/index.cfm?TopicNam (accessed 15 May 2011)
Booth, B. and MacDonald, A., 2006. ArcGIS 9: Building a Geodatabase, ESRI.

http://webhelp.esri.com/arcgisdesktop/9.2/pdf/Building_Geodat abases_Tutorial.pdf (accessed 15 May 2011)

Geometry Engine - Open Source.www.geos.osgeo.org (accessed 15 May 2011)

International Organization for Standardization, 2003. ISO 19107:2003 - Spatial schema

International Organization for Standardization. www.iso.org (access 15 May 2011)

Karimi, H. \& Akinci, B. 2010. CAD and GIS Integration. London - New York: CRC Press, Taylor \& Francis Group.

Open Geospatial Consortium, Herring J. R (ed), 2010. OpenGIS Implementation Standard for Geographic information - Simple feature access - Part 1: Common architecture.

Open Geospatial Consortium. http://www.opengeospatial.org/ (access 15 May 2011)

Pantazis, D, 1994. "Analyse méthodologique des phases de conception et de développement d'un système d'information géographique. Une proposition de méthode de conception de S.I.G. et un exemple réel de son application. Le cas du Ministère de l'Aménagement du Territoire du Grand - Duché de Luxembourg.», Ph.D..Thesis, Geography Department, University of Liege, Belgium.

Pantazis, D., Donnay J.P. 1996. La conception de SIG, Méthode et formalisme, Hermès, Paris, 343 p. (monograph).

Pantazis, D., 1997, CON.G.O.O. : A conceptual formalism for geo-graphic database design, chapter 23 of the book «Geographic Information Research : Bridging the Atlantic », Max Craglia, Helen Couclelis (Eds), Taylor and Francis, London, p. 341-359

Quantum GIS. www.qgis.org (access 15 May 2011) 